\title{
Alterações Transitórias do Exame Neurológico Durante o Despertar da Anestesia com Enflurano, Isoflurano ou Sevoflurano *
}

\section{Transient Neurological Changes During Emergence from Enflurane, Isoflurane or Sevoflurane Anesthesia}

Luiz Fernando Soares ${ }^{1}$, Pablo Escovedo Helayel ${ }^{1}$, Getúlio Rodrigues de Oliveira Filho, TSA ${ }^{2}$, Rogério do Amaral ${ }^{3}$

\section{RESUMO}

Soares LF, Helayel PE, Oliveira Filho GR, Amaral R - Alterações Transitórias do Exame Neurológico Durante o Despertar da Anestesia com Enflurano, Isoflurano ou Sevoflurano

Justificativa e Objetivos - Anormalidades transitórias do exame neurológico ocorrem durante o despertar da anestesia com halotano, enflurano e isoflurano. Pouco se conhece sobre a ocorrência de anormalidades do exame neurológico durante a recuperação da anestesia com sevoflurano. Este estudo teve como objetivo comparar a prevalência de tais achados durante a recuperação da anestesia com enflurano (Grupo E), isoflurano (Grupo I) e sevoflurano (Grupo S).

Método - Foram estudados 44 pacientes que receberam anestesia com enflurano, isoflurano ou sevoflurano em $\mathrm{N}_{2} \mathrm{O}$ a $50 \%$. Foram anotados antes da indução, imediatamente após a cessação da administração do anestésico e 5, 10, 15, 20, 30 e 40 minutos após: temperatura timpânica, nível de consciência, tônus muscular, reflexos pupilar, ciliar, bicipital, patelar e cutâneo-plantar, bem como a ocorrência de calafrios.

Resultados - As respostas dos reflexos pupilar, ciliar, patelare cutâneo-plantar correlacionaram-se com o nível de consciência. Os grupos não diferiram quanto à prevalência de hipertonia muscular, hiperreflexia bicipital, clônus plantar e resposta extensora cutâneo-plantar. Hiperreflexia patelar foi mais freqüente no grupo do enflurano do que no grupo do isoflurano. Calafrios foram mais freqüentes nos grupos $E$ e I do que no grupo do sevoflurano. A temperatura timpânica não diferiu entre os pacientes que apresentaram ou não calafrios.

Conclusões - Alterações reversíveis do exame neurológico podem estar presentes por até 40 minutos durante a recuperação da anestesia com enflurano, isoflurano ou sevoflurano.

UNITERMOS: ANESTÉSICOS, Inalatórios: enflurano, isoflurano, sevoflurano; RECUPERAÇÃO PÓS-ANESTÉSICA

\footnotetext{
* Recebido do (Received from) Hospital Governador Celso Ramos, CET/SBA Integrado de Anestesiologia da SES-SC, Florianópolis, SC

1. Anestesiologista do Hospital Governador Celso Ramos

2. Responsável pelo CET/SBA do SES-SC

3. $M E_{2}$ do $C E T / S B A$ do SES-SC
}

Apresentado (Submitted) em 22 de fevereiro de 2001

Aceito (Accepted) para publicação em 02 de maio de 2001

Correspondência para (Mail to):

Dr. Getúlio Rodrigues de Oliveira Filho

Rua Luiz Delfino 111/902

88015-360 Florianópolis, SC

E-mail:grof@th.com.br

(C) Sociedade Brasileira de Anestesiologia, 2001

\section{SUMMARY}

Soares LF, Helayel PE, Oliveira Filho GR, Amaral R - Transient Neurological Changes During Emergence from Enflurane, Isoflurane or Sevoflurane Anesthesia

Background and Objectives - Transient neurological findings are seen during emergence from halothane, enflurane, and isoflurane anesthesia. Little is known about neurological changes during recovery from sevoflurane anesthesia. This study was aimed at comparing the incidence of such findings during recovery from enflurane (Group E), isoflurane (Group I) and sevoflurane (Group S) anesthesia.

Methods - Forty four patients were assigned to receive enflurane, isoflurane or sevoflurane anesthesia in $50 \% \mathrm{~N}_{2} \mathrm{O}$. Evaluated parameters were: tympanic temperature, consciousness level, muscle tone, pupillary, eyelash, bicipital, patellar and plantar reflexes and shivering, which were recorded before induction, immediately after anesthesia withdrawal and at 5 , $10,15,20,30$ and 40 minutes thereafter.

Results - Pupillary, eyelash, patellar and plantar reflexes were significantly related to the level of consciousness. Groups did not differ regarding the incidence of increased muscle tone, bicipital response, plantar clonus and extension plantar response. Increased patellar response was more frequent in group $E$ than in group I. Shivering was more frequent in groups $E$ and I as compared to group S. No difference in tympanic temperature could be detected among patients with or without shivering.

Conclusions - Transient neurological changes can be detected up to 40 minutes during emergence from enflurane, isoflurane or sevoflurane anesthesia.

KEY WORDS: ANESTHETICS, Inhalational: enflurane, isoflurane, sevoflurane; POSTANESTHETIC RECOVERY

\section{INTRODUÇÃO}

$\mathrm{D}$ urante o despertar da anestesia com halotano, enflurano e isoflurano, podem ser observadas alterações do exame neurológico, que se caracterizam por sinais de desinibição central das vias motoras. Assim, é possível encontrar respostas exacerbadas de reflexos monossinápticos e atividade tônico-clônica ${ }^{1,2}$. Embora sem relevância clínica, pelo seu caráter transitório, estas manifestações fazem parte do conjunto de sinais que caracteriza o despertar da anestesia inalatória e são facilmente observáveis através de um exame neurológico sumário. Portanto, seu conhecimento tem importância didática. Não são conhecidas as manifestações neurológicas que ocorrem durante o despertar da anestesia com o sevoflurano. 
Este estudo teve como objetivo descrever e comparar as alterações do exame neurológico, durante a fase de emergência da anestesia inalatória com enflurano, isoflurano e sevoflurano.

\section{MÉTODO}

Com aprovação da Comissão de Ética Médica do Hospital Governador Celso Ramos, foram incluídos no estudo 44 pacientes adultos de ambos os sexos, estado físico ASA I ou II, escalados para cirurgias eletivas sob anestesia geral. Não foram incluídos pacientes submetidos a cirurgias intracranianas ou portadores de anormalidades neurológicas. Os pacientes não receberam nenhuma medicação pré-anestésica e foram designados, por sorteio, para os seguintes grupos: grupo do enflurano $(n=14)$, anestesia inalatória com $\mathrm{N}_{2} \mathrm{O} / \mathrm{O}_{2}$ a $50 \%$ e enflurano; grupo do isoflurano $(n=15)$, anestesia inalatória com $\mathrm{N}_{2} \mathrm{O} / \mathrm{O}_{2}$ a $50 \%$ e isoflurano; ou, grupo do sevoflurano $(n=15)$, anestesia inalatória com $\mathrm{N}_{2} \mathrm{O} / \mathrm{O}_{2}$ a $50 \%$ e sevoflurano. Os pacientes foram monitorizados com eletrocardiograma (MC5), monitor não invasivo de pressão arterial, oxímetro de pulso, capnógrafo, analisador de gases anestésicos e monitor de função neuromuscular por acelerometria. A anestesia constou de indução com propofol (2 $\left.\mathrm{mg} \cdot \mathrm{kg}^{-1}\right)$, succinilcolina $\left(1,5 \mathrm{mg} \cdot \mathrm{kg}^{-1}\right)$ e intubação orotraqueal. Atracúrio ou vecurônio foram administrados durante o procedimento, caso necessários. A função neuromuscular foi revertida com neostigmina $\left(35 \mathrm{a} 50 \mu \mathrm{g} \cdot \mathrm{kg}^{-1}\right)$, precedida de atropina $\left(20 \mu \mathrm{g} \cdot \mathrm{kg}^{-1}\right)$, até obter relação T4/T1 da seqüência de quatro estímulos igual ou maior que 0,9 . A concentração inspirada dos anestésicos inalatórios foi ajustada para manter a pressão arterial sistólica e a freqüência cardíaca entre mais ou menos $20 \%$ dos valores pré-anestésicos.

Os seguintes parâmetros foram avaliados imediatamente antes da indução anestésica (MP), ao final da anestesia, antes de cessada a administração dos agentes inalatórios (M0) e 5, 10, 15, 20, 30 e 40 minutos após cessada a administração do anestésico inalatório (M5 a M40): nível de consciência (4 = lúcido, 3 = sonolento, desperta ao comando verbal, 2 = sonolento, desperta ao toque, 1 = agitado, desorientado $0=$ coma), reflexo fotomotor ( $1=$ presente, $0=$ ausente), reflexo ciliar ( $1=$ presente, $0=$ ausente), tônus muscular ( $3=$ aumentado, 2 = normal, 1 = diminuído, $0=$ ausente), reflexo bicipital ( 3 = aumentado, 2 = normal, 1 = diminuído, 0 = ausente), reflexo patelar ( 3 = aumentado, 2 = normal, 1 = diminuído, $0=$ ausente), pesquisa de clônus plantar $(2=$ sustentado por mais de 5 segundos, 1 = sustentado por cinco segundos ou menos, 0 = ausente), reflexo cutâneo-plantar ( 2 = resposta extensora, 1 = resposta flexora, $0=$ ausente), reação ao tubo traqueal $(1=$ presente, $0=$ ausente $)$, calafrios $(1=$ presentes, $0=$ ausentes).

As avaliações foram realizadas por observador que desconhecia o anestésico inalatório utilizado no paciente. Para tal, os vaporizadores foram cobertos e o observador retirou-se da sala cirúrgica após realizada a avaliação inicial, retornando somente após cessada a administração do anestésico. As variáveis antropométricas foram comparadas, entre os grupos, por análise de variância unifatorial e pelo teste do Qui-quadrado. Os percentuais de anormalidades neurológicas durante o período de observação foram comparados, entre os grupos, pelos testes do Qui-quadrado e exato de Fisher. Coeficientes R de Spearman foram calculados entre os escores de cada parâmetro do exame neurológico e os escores de consciência. A temperatura timpânica entre os pacientes que apresentaram e os que não apresentaram calafrios foi comparada pelo teste $t$ de Student. O nível de significância foi de $5 \%$.

\section{RESULTADOS}

Não houve diferenças demográficas e nem quanto à duração da anestesia entre os grupos (Tabela I).

A consciência foi recuperada mais precocemente pelos pacientes do grupo do sevoflurano (Figura 1). Não houve diferença entre os grupos quanto ao retorno da resposta normal dos reflexos fotomotor e ciliar (Figuras 2 e 3). Exceto pela menor prevalência no grupo do sevoflurano, em relação ao grupo do enflurano, em M20, não houve diferença significativa entre os grupos quanto à ocorrência de hipertonia muscular (Figura 4). Os pacientes do grupo do enflurano apresentaram maior prevalência de hiperreflexia patelar do que os do grupo do isoflurano de M10 a M40 (Figura 5). Clônus plantar foi mais freqüente no grupo do enflurano dos que nos grupos I e S em M15, M20 e M40. Em M30 somente foi detectada diferença significativa entre os grupos E e I (Figura 6). Os pacientes do grupo do sevoflurano apresentaram reposta cutâneo-plantar extensora somente em M5. Apesar disto, não houve diferença significativa entre os percentuais de resposta cutâneo-plantar extensora dos outros grupos ou quando comparados ao grupo do sevoflurano (Figura 7). Não houve diferenças entre os grupos quanto à ocorrência de hiperreflexia bicipital (Figura 8).

Tabela I - Dados Demográficos

\begin{tabular}{lccc}
\hline & Grupo E & Grupo I & Grupo S \\
\hline Idade (anos) * & $31,7 \pm 10,4$ & $34,5 \pm 14,6$ & $36,2 \pm 14,3$ \\
Sexo (M/F) ** & $9 / 5$ & $8 / 7$ & $8 / 7$ \\
Peso $(\mathrm{kg})^{*}$ & $67,5 \pm 10,7$ & $71,4 \pm 12,2$ & $67,1 \pm 11,9$ \\
Altura $(\mathrm{m}){ }^{*}$ & $1,70 \pm 0,08$ & $1,71 \pm 0,07$ & $1,65 \pm 0,06$ \\
Duração da anestesia (minutos) ${ }^{*}$ & $118,9 \pm 79,7$ & $108,2 \pm 56,8$ & $101 \pm 58,1$ \\
\hline
\end{tabular}

* Dados representados pela Média \pm DP; ** Dados representados pelas freqüências 


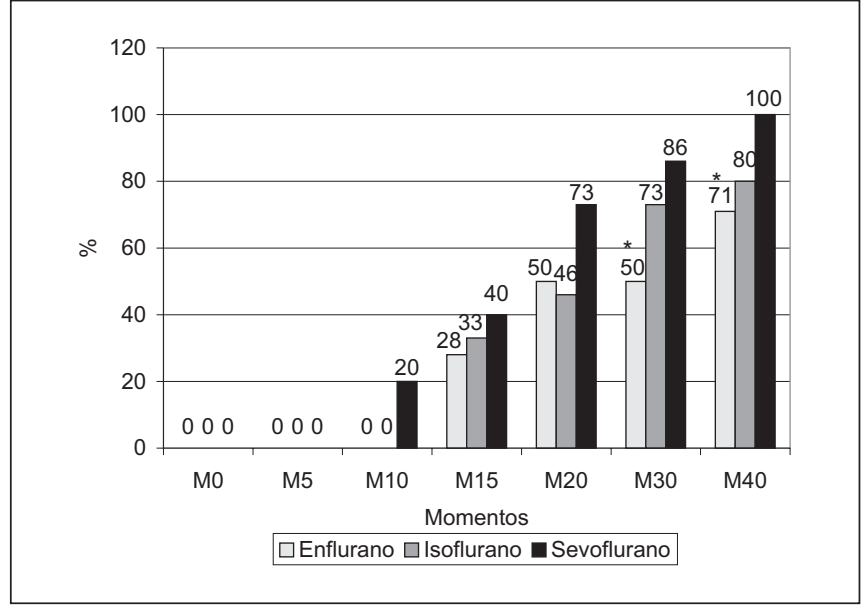

Figura 1 - Percentuais de Pacientes Apresentando Consciência Totalmente Recuperada em Cada Momento Após o Término da Anestesia

${ }^{*}=p<0,05$ comparado ao grupo $S$

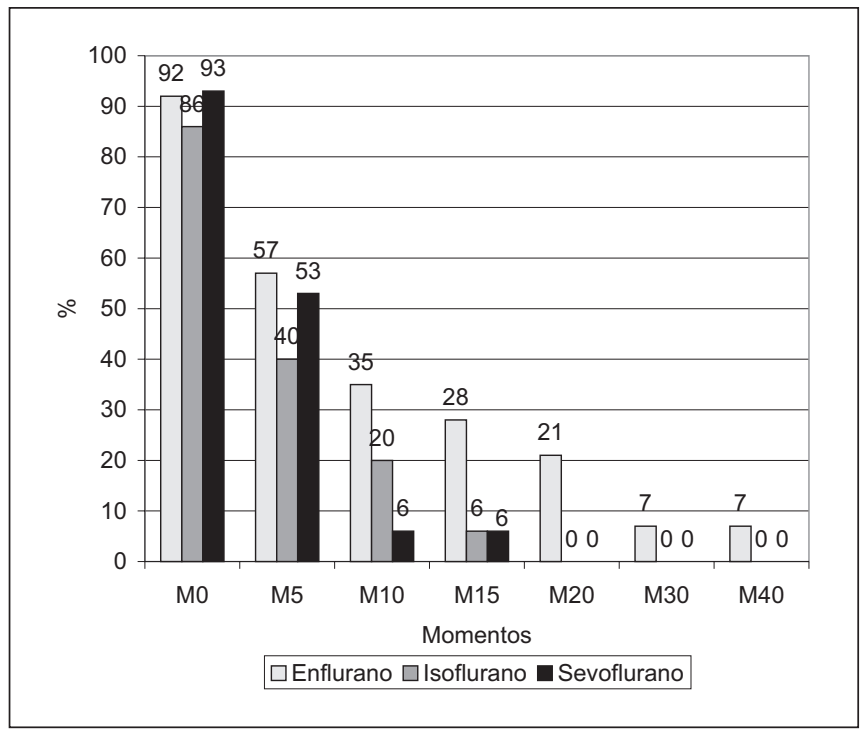

Figura 2 - Percentuais de Pacientes Apresentando Ausência do Reflexo Fotomotor em Cada Momento Após o Término da Anestesia

Foram observados calafrios nos pacientes do grupo do sevoflurano apenas em M20. A prevalência de calafrios foi significativamente maior nos grupos E e I do que no grupo do sevoflurano, somente em M30. Em M40 somente o grupo do enflurano diferiu significativamente do grupo do sevoflurano (Figura 9). Atemperatura timpânica dos pacientes que apresentaram calafrios foi de $35,19 \pm 0,61^{\circ} \mathrm{C}$ e dos que não apresentaram foi de $35,46 \pm 0,9^{\circ} \mathrm{C}(p=0,33)$.

As prevalências de anormalidades do exame neurológico que ocorreram em todo o período de observação estão representadas na tabela II. Houve diferença significativa entre a prevalência de hiperreflexia patelar no grupo do enflurano, comparada à do grupo do sevoflurano. Calafrios foram mais prevalentes nos grupos $\mathrm{E}$ e I do que no grupo do sevoflurano.

Revista Brasileira de Anestesiologia

Vol. 51, № 6, Novembro - Dezembro, 2001

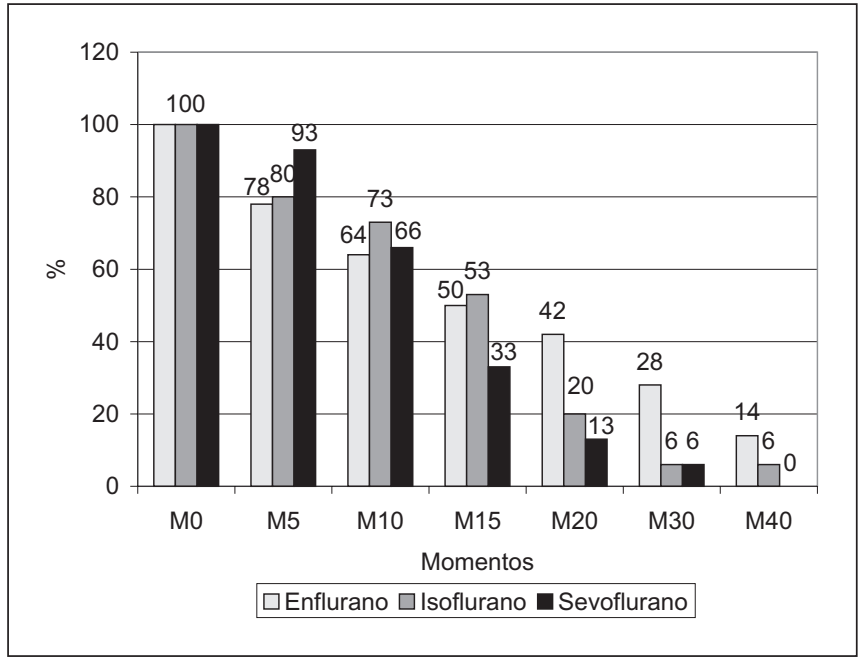

Figura 3 - Percentuais de Pacientes Apresentando Ausência do Reflexo Ciliar em Cada Momento Após o Término da Anestesia

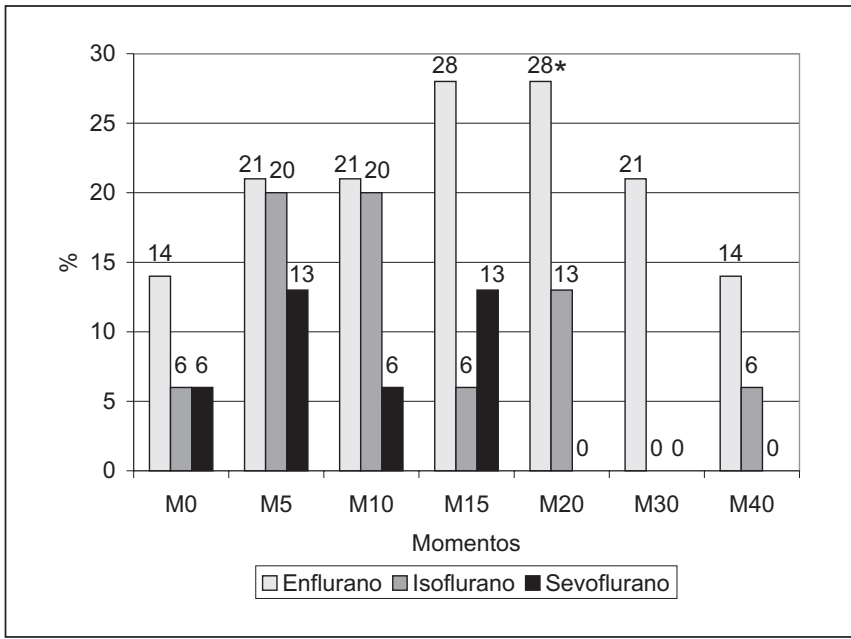

Figura 4 - Percentuais de Pacientes Apresentando Hipertonia Muscular em Cada Momento Após o Término da Anestesia

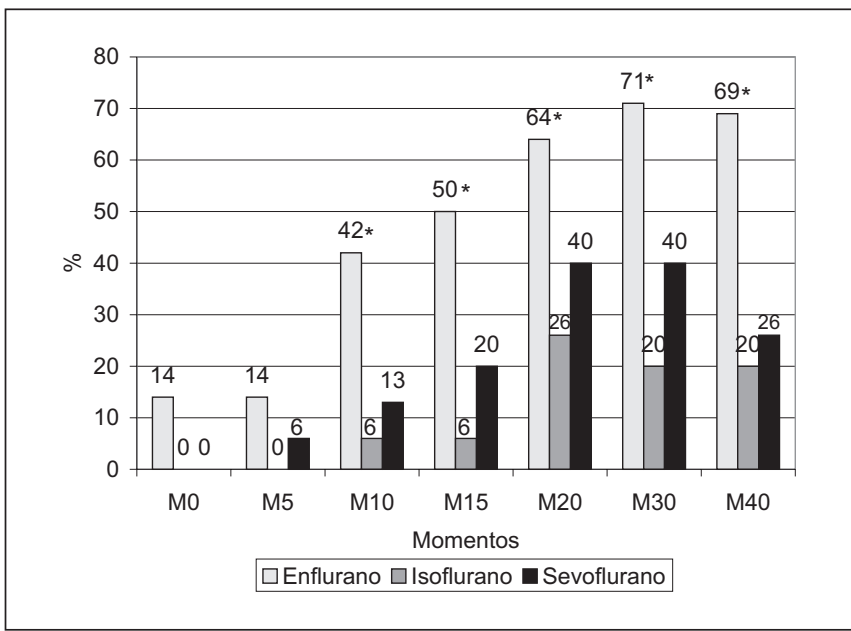

Figura 5 - Percentuais de Pacientes Apresentando Hiperreflexia Patelar em Cada Momento Após o Término da Anestesia $* p<0,05$ comparado ao grupo 1 


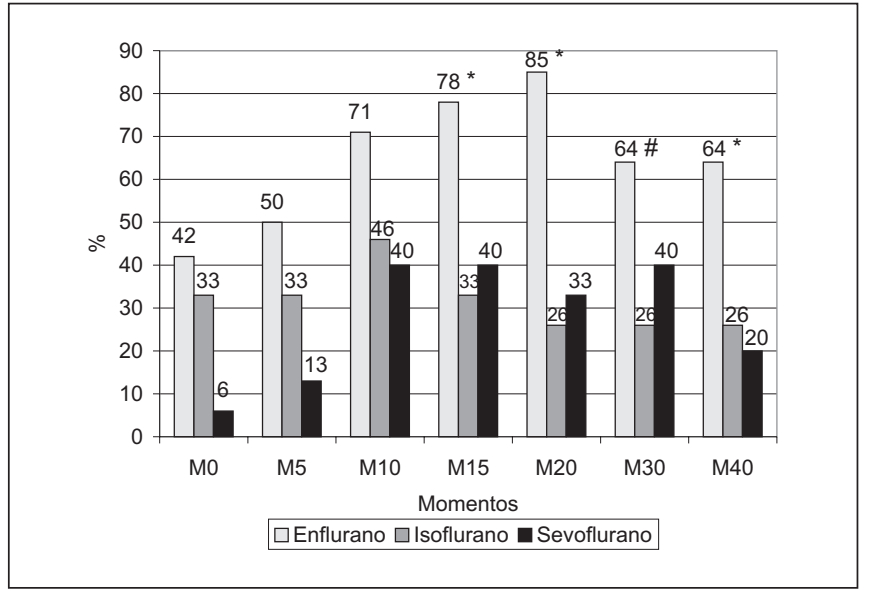

Figura 6 - Percentuais de Pacientes Apresentando Clônus Plantar Sustentado ou Não em Cada Momento Após o Término da Anestesia

${ }^{*} p<0,05$ comparado aos grupos I e $S$

$\# p<0,05$ comparado ao grupo I

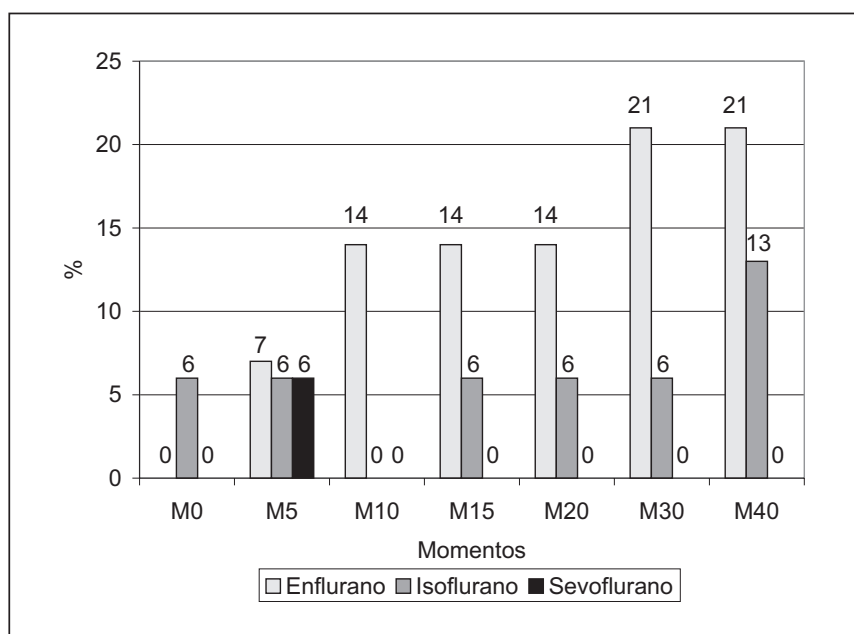

Figura 7 - Percentuais de Pacientes Apresentando Resposta Cutâneo-Plantar Extensora em Cada Momento Após o Término da Anestesia

O retorno à normalidade dos reflexos fotomotor, ciliar, patelar e cutâneo plantar correlacionou-se significativamente com o retorno da consciência (coeficientes $\mathrm{R}$ de Spearman $=$ $0,61,0,79,0,23$ e 0,11 , respectivamente).

Tabela II - Percentuais de Pacientes Apresentando Alterações Neurológicas Durante o Período de Observação

\begin{tabular}{lccc}
\hline & Grupo E & Grupo I & Grupo S \\
\hline Hipertonia muscular & 57 & 40 & 27 \\
Hiperreflexia bicipital & 21 & 20 & 20 \\
Hiperreflexia patelar & $79^{*}$ & 27 & 47 \\
Clônus plantar & 86 & 53 & 67 \\
Resposta cutâneo-plantar extensora & 29 & 27 & 7 \\
Calafrios & $50^{* *}$ & 40 & 6 \\
\hline
\end{tabular}

* $=p<0,05$ comparado ao grupo I

${ }^{* *}=p<0,05$ comparado ao grupo $\mathrm{S}$

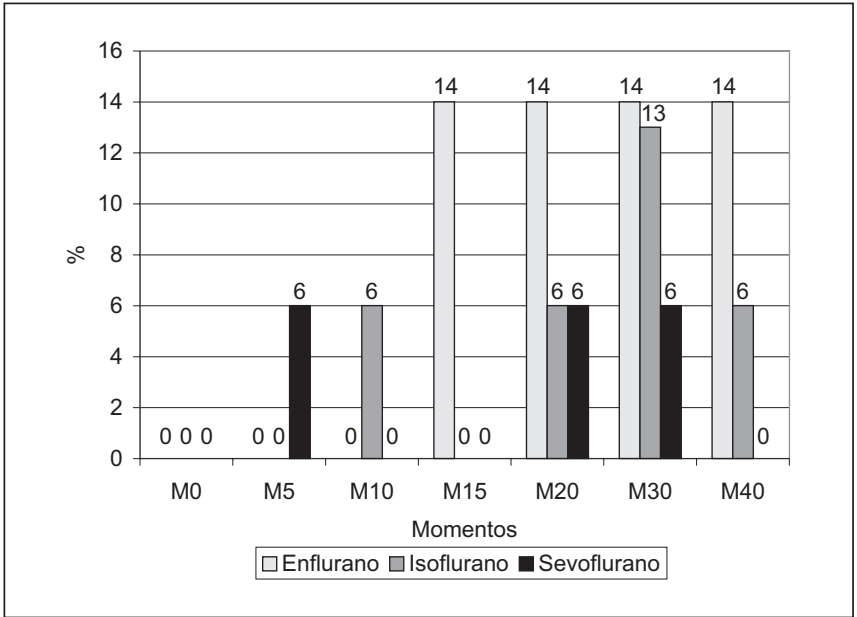

Figura 8 - Percentuais de Pacientes Apresentando Hiperreflexia bicipital em Cada Momento Após o Término da Anestesia

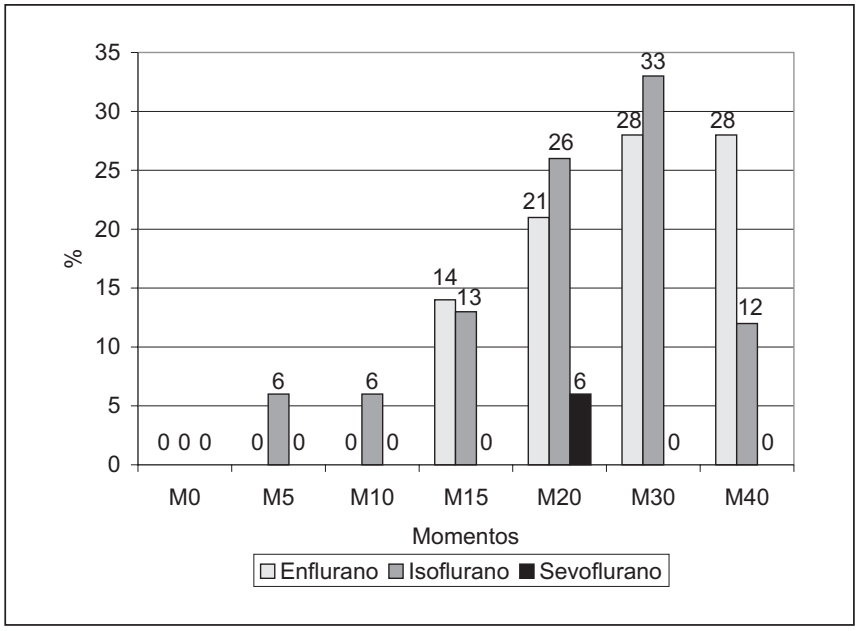

Figura 9 - Percentuais de Pacientes Apresentando Calafrios em Cada Momento Após o Término da Anestesia

\section{DISCUSSÃO}

Resposta extensora cutâneo-plantar, hipertonia muscular, hiperreflexia bicipital e patelar e clônus plantar representam lesão das vias piramidais ${ }^{3}$. Contudo, estes reflexos também podem ocorrer durante o período pós-anestésico imediato, sem representar dano neurológico permanente ${ }^{1,2}$. Geralmente estes sinais neurológicos anormais não persistem por mais de 60 minutos $^{2}$, ao contrário da psicomotricidade ${ }^{4}$ e do teste da apnéia após hiperventilação ${ }^{5}$, que podem persistir alterados por até 120 minutos após o término da anestesia.

Neste estudo, o grupo do enflurano apresentou uma prevalência significativamente maior de hiperreflexia patelar, quando comparada ao grupo do isoflurano. Outros autores também relatam que a anestesia com enflurano resulta em maior prevalência de anormalidades neurológicas transitórias que anestesias realizadas com halotano ${ }^{1}$ ou isoflurano ${ }^{2}$,

Revista Brasileira de Anestesiologia Vol. 51, № 6 , Novembro - Dezembro, 2001 
embora outro estudo comparando o enflurano com isoflurano, não tenha encontrado diferença significativa entre a prevalência de achados neurológicos anormais entre os dois agentes ${ }^{4}$. Entretanto, o grupo do sevoflurano não diferiu dos demais, quanto à prevalência de hiperreflexia patelar.

O mecanismo exato destes achados ainda é desconhecido, mas poderia ser resultado da recuperação em tempos diferentes entre os vários centros do sistema nervoso central $(\mathrm{SNC})^{6}$. Estes sinais de desinibição de neurônio motor podem ser causados pela recuperação do sistema reticular ativador precedendo a dos centros corticais, já que as vias inibitórias desse sistema dependem de impulsos de centros corticais, enquanto as células facilitatórias têm sua própria atividade espontânea. Desta forma, durante a recuperação da anestesia, é possível que exista um período de hiperexcitabilidade das células do corno anterior da medula espinhal, o que poderia explicar estes achados.

Alternativamente um efeito excitatório direto sobre o SNC poderia ser responsável por estas anormalidades. Em um estudo com camundongos anestesiados com isoflurano, observou-se que o processamento de dopamina aumenta significativamente nos gânglios da base e que este achado pode ser responsabilizado pela hiperlocomoção dos animais, observada no período de recuperação da anestesia ${ }^{7}$.

Neste estudo, a prevalência total de calafrios foi significativamente maior nos grupo do enflurano e do isoflurano do que no do sevoflurano. Contudo, não se observou diferença entre a temperatura timpânica dos pacientes que apresentaram e a dos que não apresentaram calafrios pós-anestésicos. Em outro estudo ${ }^{8}$, ocorreram calafrios em pacientes anestesiados com isoflurano que se apresentaram normotérmicos ao término da anestesia, embora $80 \%$ apresentassem vasoconstrição periférica termorregulatória. Os autores sugerem que o tremor pode ter origem multifatorial, dependendo da temperatura e do tipo de anestesia. Estudos realizados com eletromiografia sugerem que existem pelo menos dois tipos diferentes de tremores, um com padrão tônico que lembra o calafrio termorregulatório normal, e outro com padrão clônico que aparece especificamente na recuperação de anestésicos voláteis ${ }^{9}$. É possível que este padrão específico de tremor possa resultar da desinibição do controle normal sobre os reflexos espinhais, induzida pelo anestési$\mathrm{Co}^{8,9}$

Estudos prévios demonstram que o retorno à consciência correlaciona-se com o retorno à normalidade dos reflexos pupilar e ciliar ${ }^{1,2}$. Neste estudo, além de correlacionar-se com retorno à normalidade dos reflexos pupilar e ciliar, a consciência também se correlacionou com o desaparecimento de hiperreflexia patelar e da resposta extensora do reflexo cutâneo-plantar.

Conclui-se que o enflurano, o isoflurano e o sevoflurano causam alterações neurológicas transitórias qualitativamente semelhantes, durante o despertar da anestesia, embora o enflurano cause maior prevalência de hiperreflexia patelar que o isoflurano. Calafrios ocorrem mais freqüentemente durante a emergência da anestesia com enflurano e isoflurano. Assim como em estudos precedentes ${ }^{1,2}$, as anormalida- des do exame neurológico são mais evidentes nos membros inferiores. Vale ressaltar que, neste estudo, as alterações neurológicas reverteram em 40 minutos.

\section{Transient Neurological Changes During Emergence from Enflurane, Isoflurane or Sevoflurane Anesthesia}

Luiz Fernando Soares, M.D., Pablo Escovedo Helayel, M.D., Getúlio Rodrigues de Oliveira Filho, M.D., Rogério do Amaral, M.D.

\section{INTRODUCTION}

Neurological changes can be seen during emergence from halothane, enflurane and isoflurane and are characterized by signs of central desinhibition of motor pathways. So, it is possible to find exacerbated responses to monosympathetic reflexes and tonico-clonic activity ${ }^{1,2}$. These transient manifestations are clinically irrelevant, but are part of a set of signs characterizing inhalational anesthesia emergence which are easily detected by a brief neurological exam, thus being didactically important. Neurological manifestations during emergence from sevoflurane anesthesia are not known. The aim of this study was to describe and to compare neurological changes during emergence from inhalational enflurane, isoflurane and sevoflurane anesthesia.

\section{METHODS}

After Hospital Governador Celso Ramos Medical Ethics Committee approval, participated in this study 44 adult patients of both genders, physical status ASA I or II scheduled for elective surgeries under general anesthesia. Patients submitted to intracranial surgeries or with neurological disorders were excluded from this study. Patients were given no premedication and were allocated into the following groups: enflurane group $(n=14)$ - inhalational anesthesia with $50 \% \quad \mathrm{~N}_{2} \mathrm{O} / \mathrm{O}_{2}$ and enflurane; isoflurane group $(\mathrm{n}=15)$ inhalational anesthesia with $50 \% \mathrm{~N}_{2} \mathrm{O} / \mathrm{O}_{2}$ and isoflurane; and sevoflurane group $(n=15)$ - inhalational anesthesia with $50 \%$ $\mathrm{N}_{2} \mathrm{O} / \mathrm{O}_{2}$ and sevoflurane. Patients were monitored with ECG (MC5), non-invasive blood pressure, pulse oximetry, capnography, anesthetic gas analyzer and neuromuscular function monitor. Anesthesia was induced with propofol (2 $\left.\mathrm{mg} \cdot \mathrm{kg}^{-1}\right)$, succinylcholine $\left(1.5 \mathrm{mg} \cdot \mathrm{kg}^{-1}\right)$ followed by tracheal intubation. Atracurium and vecuronium were administered during the procedure, as needed. Neuromuscular function was reverted with neostigmine ( 35 to $50 \mu \mathrm{g} \mathrm{kg}^{-1}$ ), preceded by atropine $\left(20 \mu \mathrm{g} \cdot \mathrm{kg}^{-1}\right)$ until T4/T1 ratio of TOF stimulation was equal to or higher than 0.9 . Inhalational anesthetic inspired concentration was adjusted to maintain systolic blood pressure and heart rate within approximately $20 \%$ of preanesthetic values. 
The following parameters were evaluated immediately before induction (MP), at the end of anesthesia before withdrawing inhalational agents (M0), and at 5, 10, 15, 20, 30 and 40 minutes thereafter (M5 to M40): consciousness level (4 = conscious, 3 = somnolent, awakens with verbal command, 2 = somnolent, wakens when touched, 1 = agitated, disoriented, $0=$ comma), photomotor reflex $(1=$ present, $0=$ absent $)$, eyelash reflex $(1=$ present, $0=$ absent $)$, muscle tone $(3$ = increased, $2=$ normal, $1=$ decreased, $0=$ absent $)$, bicipital reflex $(3=$ increased, $2=$ normal, $1=$ decreased, $0=$ absent $)$, patellar reflex $(3=$ increased, $2=$ normal, $1=$ decreased, $0=$ absent), plantar clonus ( 2 = sustained for more than 5 seconds, 1 = sustained for 5 seconds or less, $0=$ absent $)$, plantar reflex $(2=$ extension response, $1=$ flexion response, $0=a b$ sent), reaction to tracheal tube ( 1 = present, 2 = absent $)$, shivering $(1=$ present, $0=$ absent $)$.

Evaluations were performed by an observer blind to the inhalational anesthetic drug. For such, vaporizers were covered and the observer left the operating room after initial evaluation returning only after anesthesia withdrawal.

Demographics data were compared between groups by unifactorial analysis of variance and Chi-square test. The incidence of neurological abnormalities was compared between groups by Chi-square and Fisher exact tests. Spearman R coefficients were calculated between scores of each neurological parameters and consciousness scores. Tympanic temperature of patients with or without shivering was compared by Student's $t$ test. Significance level was stablished to $5 \%$.

\section{RESULTS}

There were no difference in demographics data or anesthesia duration between groups (Table I).

Sevoflurane group patients (Figure 1) had an earlier recovery. There was no difference between groups in times to return to normal photomotor and eyelash reflexes (Figures 2 and 3 ). There was no significant difference between groups in muscle hypertonia in $M 20$, except for its lower incidence in sevoflurane group compared to enflurane group (Figura 4). Enflurane group patients presented a higher incidence of increased patellar reflex as compared to isoflurane group patients from M10 to M40 (Figure 5). Plantar clonus was more frequent in the enflurane group as compared to groups I and $S$ in M15, M20 and M40. There was a significant difference in M30 only between groups E and I (Figure 6). Sevoflurane

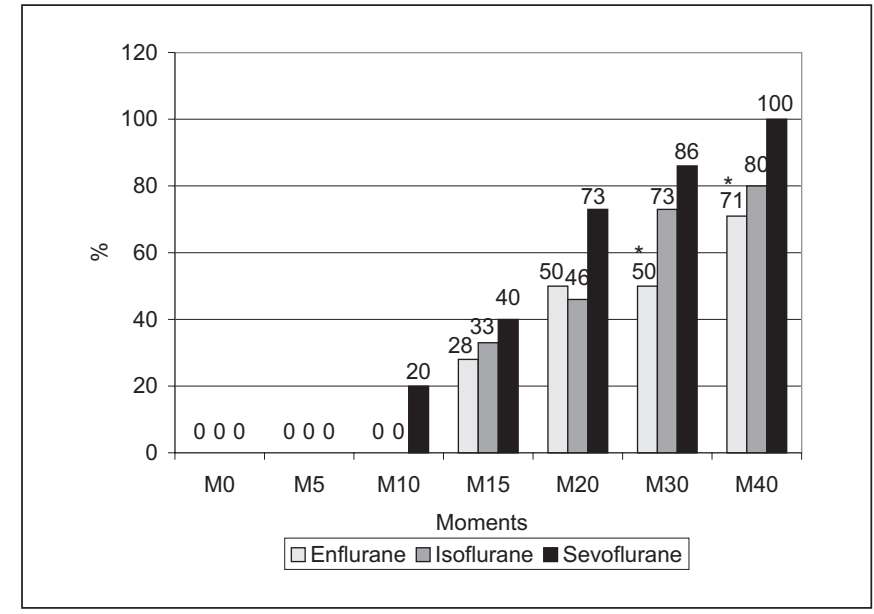

Figure 1 - Patients Totally Conscious at Every Moment After Anesthesia Withdrawal (\%)

${ }^{*}=p<0.05$ as compared to group $S$

Table I - Demographics Data

\begin{tabular}{lccc}
\hline & Group E & Group I & Group S \\
\hline Age $($ years) * & $31.7 \pm 10.4$ & $34.5 \pm 14.6$ & $36.2 \pm 14.3$ \\
Gender $(\mathrm{M} / \mathrm{F}){ }^{* *}$ & $9 / 5$ & $8 / 7$ & $8 / 7$ \\
Weight $(\mathrm{kg})^{*}$ & $67.5 \pm 10.7$ & $71.4 \pm 12.2$ & $67.1 \pm 11.9$ \\
Height $(\mathrm{m})^{*}$ & $1.70 \pm 0.08$ & $1.71 \pm 0.07$ & $1.65 \pm 0.06$ \\
Anesthesia duration (minutes) * & $118.9 \pm 79.7$ & $108.2 \pm 56.8$ & $101 \pm 58.1$ \\
\hline
\end{tabular}

* Data expressed in Mean \pm SD; ** Data represented by frequency 


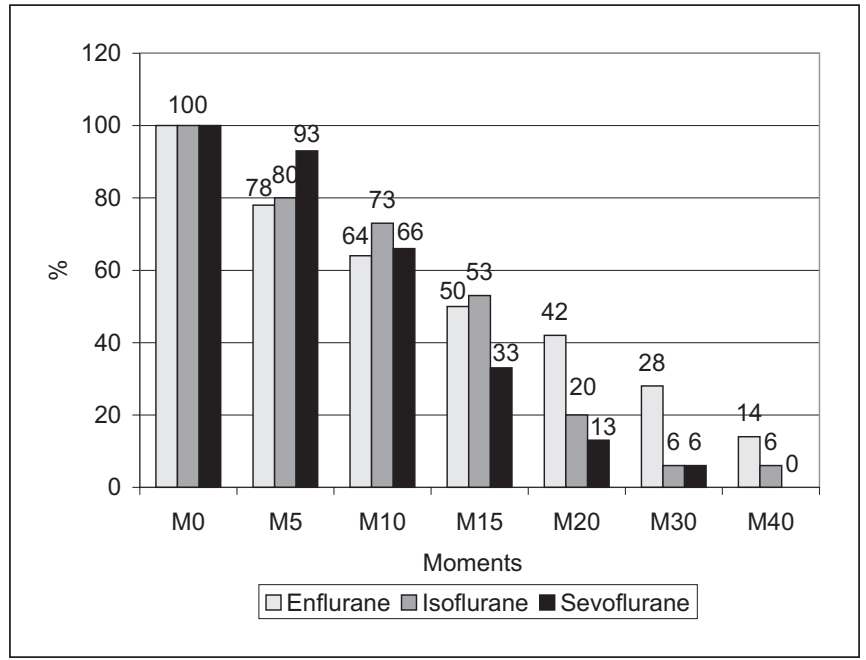

Figure 3 - Patients without Eyelash Reflex at Each Moment After Anesthesia Withdrawal (\%)

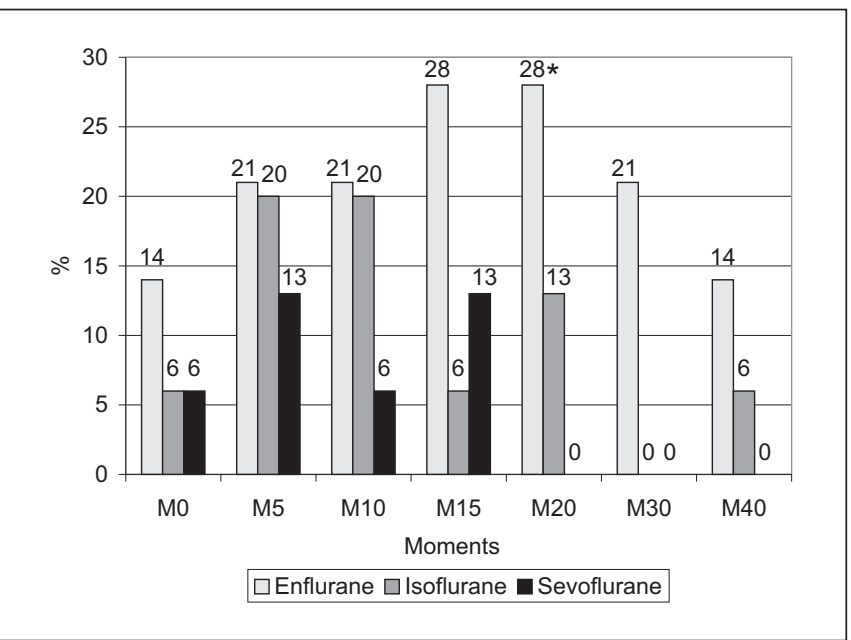

Figure 4 - Patients with Increased Muscle Tone at Each Moment After Anesthesia Withdrawal (\%)

$*=p<0.05$ as compared to group $S$

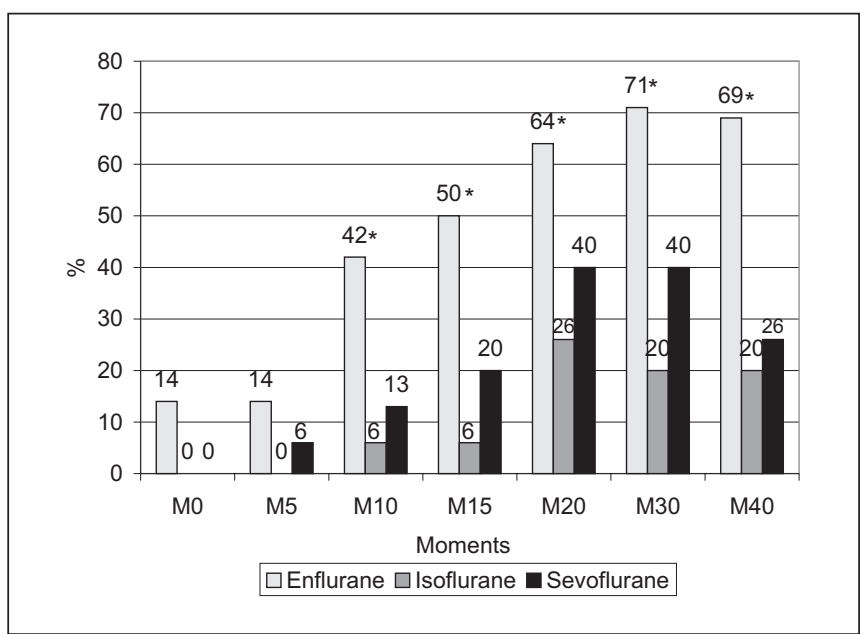

Figure 5 - Patients with Increased Patellar Reflex at Each Moment After Anesthesia Withdrawal (\%)

${ }^{*} p<0.05$ as compared to group I

Revista Brasileira de Anestesiologia

Vol. 51, № 6, Novembro - Dezembro, 2001

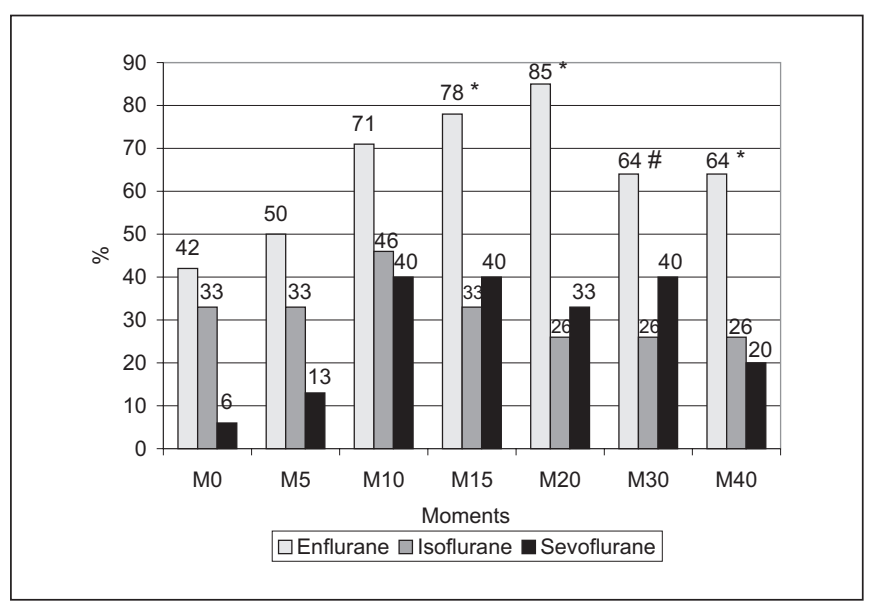

Figure 6 - Patients with Sustained or Not Sustained Plantar Clonus at Each Moment After Anesthesia Withdrawal (\%) ${ }^{*} p<0.05$ as compared to groups I and $S$

$\# p<0.05$ as compared to group I

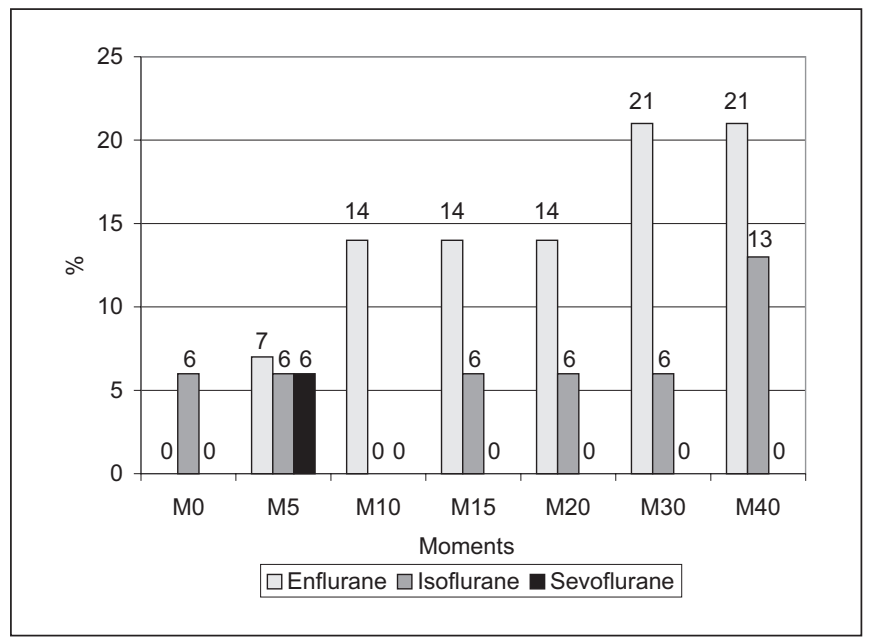

Figure 7 - Patients with Upgoing Plantar Response at Each Moment After Anesthesia Withdrawal (\%)

group patients showed extension plantar response only in M5. In spite of this, there was no significant difference in extension plantar response between other groups or when compared to the sevoflurane group (Figure 7). There was no difference between groups in increased bicipital reflex incidence (Figure 8).

Sevoflurane group patients had shivering only in M20. In $\mathrm{M} 30$, the incidence of shivering was significantly higher in groups E and I as compared to the sevoflurane group. In M40, only the enflurane group was significantly different from the sevoflurane group (Figure 9). Mean tympanic temperatures were $35.19 \pm 0.61^{\circ} \mathrm{C}$ and $35.46 \pm 0.9^{\circ} \mathrm{C}$ for patients with and without shivering respectively $(p=0.33)$.

The incidence of neurological changes throughout the observation period is shown in table II. There was a significant difference in increased patellar reflex incidence in the enflurane group as compared to the sevoflurane group. The incidence of shivering was also higher in groups $\mathrm{E}$ and $\mathrm{I}$ as compared to the sevoflurane group. 


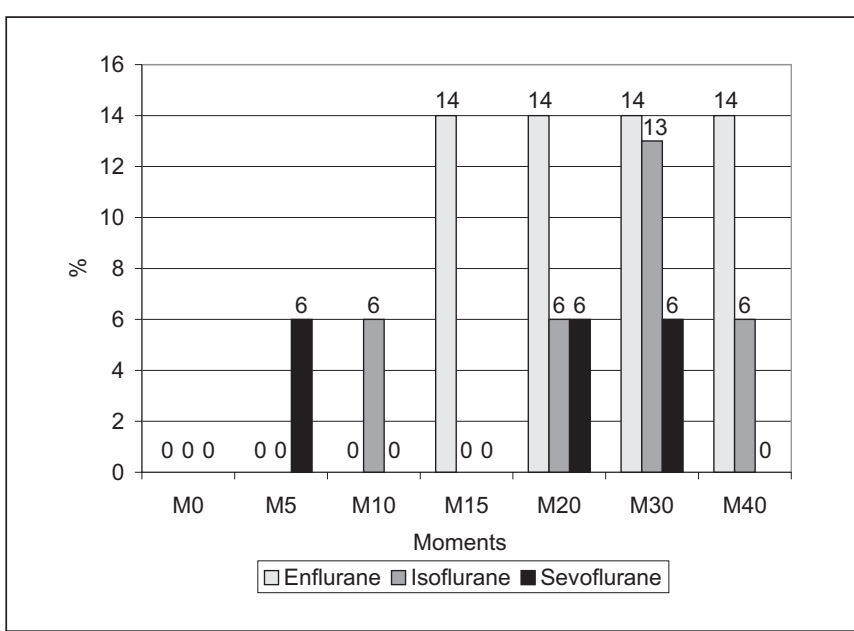

Figure 8 - Patients with Increased Biceptal Reflex at Each Moment After Anesthesia Withdrawal (\%)

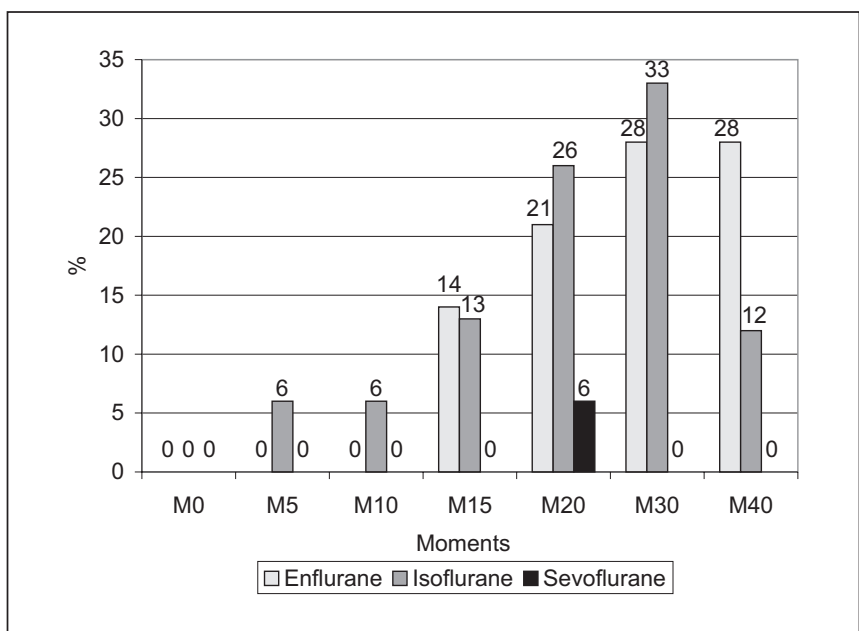

Figure 9 - Patients with Shivering at Each Moment After Anesthesia Withdrawal (\%)

Photomotor, eyelash, patellar and plantar reflexes recovery was significantly related to the consciousness recovery (Spearman R coefficients - 0.61, 0.79, 0.23 and 0.11, respectively).

Table II - Patients with Neurological Changes during the Observation Period (\%)

\begin{tabular}{lccc}
\hline & Group E & Group I & Group S \\
\hline Increased muscle tone & 57 & 40 & 27 \\
Increased bicipital response & 21 & 20 & 20 \\
Increased patellar response & $79 *$ & 27 & 47 \\
Plantar clonus & 86 & 53 & 67 \\
Upgoing plantar response & 29 & 27 & 7 \\
Shivering & $50^{* *}$ & 40 & 6 \\
\hline
\end{tabular}

* $=p<0.05$ as compared to group I

$* *=p<0.05$ as compared to group $S$

\section{DISCUSSION}

Extension plantar response, muscle hypertonia, increased bicipital and patellar reflexes as well as plantar clonus are signs of piramidal pathway injury ${ }^{3}$. However, they may also occur in the immediate postanesthetic period and not represent permanent neurological damage ${ }^{1,2}$. In general, these abnormal neurological signs do not last more than 60 minutes ${ }^{2}$, in contrast to psychomotricity ${ }^{4}$ and to the apnea test after hyperventilation ${ }^{5}$, which may persist altered for up to 120 minutes after anesthesia.

In our study, the enflurane group presented a significantly higher incidence of increased patellar reflex as compared to the isoflurane group. Other authors have also reported that enflurane results in a higher incidence of transient neurological changes as compared to halothane ${ }^{1}$ or isoflurane ${ }^{2}$, although a different study comparing enflurane with isoflurane has not found significant differences between both agents ${ }^{4}$. The sevoflurane group, however, was not different from the others regarding the increased patellar reflex incidence.

The exact mechanism of such findings is still unknown, but could be the result of different recovery rates of various CNS centers ${ }^{6}$. These motor neuronal desinhibition signs could be caused by the reticular activator system recovery preceding the recovery of cortical centers, since inhibitory pathways of such system depend on cortical center impulses while excitatory cells have their own spontaneous activity. Hence, during anesthesia recovery, there might to be a hyper-excitability period of spinal cord anterior horn cells, which could explain such findings.

On the other hand, a direct excitatory effect on the CNS could explain such abnormalities. A study with rats anesthetized with isoflurane has shown that dopamine processing is significantly increased in basal glanglia and that this finding could be the cause for animals hypermotility observed in the postanesthetic recovery period ${ }^{7}$.

In our study, the incidence of shivering was significantly higher in the enflurane and isoflurane groups as compared to the sevoflurane group. However, there was no difference in tympanic temperature when comparing patients with and without postanesthetic shivering. In a different study ${ }^{8}$, shivering was observed in patients anesthetized with isoflurane who were normothermic at the end of anesthesia, although $80 \%$ presented with thermoregulating peripheral vasoconstriction. The authors suggest that shivering could have a multifactorial origin depending on temperature and type of anesthesia. Electromyographic studies suggest the existence of at least two different types of shivering: the first with tonic pattern resembling normal thermoregulating shivering, and the other with clonic pattern, specific of volatile anesthetics recovery ${ }^{9}$. It is possible that this specific shivering pattern could result from an anesthetic-induced desinhibition of the normal control over spinal reflexes ${ }^{8,9}$.

Previous studies have shown that consciousness recovery is related to pupillary and eyelash reflexes recovery 1,2 . In our study, in addition to being related to pupillary and eyelash reVol. 51, № 6, Novembro - Dezembro, 2001 
flexes recovery, consciousness was also related to the disappearance of increased patellar reflex and extension plantar response.

The conclusion was that enflurane, isoflurane and sevoflurane induce qualitatively similar transient neurological changes during anesthesia emergence, but enflurane induces a higher incidence of increased patellar reflex as compared to isoflurane. Shivering was more frequent with enflurane and isoflurane. As in previous studies ${ }^{1,2}$, neurological abnormalities were more evident in lower limbs. It is worth stressing that, within our study, neurological changes disappeared in 40 minutes.

\section{REFERÊNCIAS - REFERENCES}

01. Rosenberg $\mathrm{H}$, Clofine R, Bialik $\mathrm{O}$ - Neurologic changes during awakening from anesthesia. Anesthesiology, 1981;54:125-130.

02. McCulloch P, Milne B - Neurological phenomena during emergence from enflurane or isoflurane anaesthesia. Can J Anaesth, 1990;37:739-742.

03. Adams RD, Victor M - Principles of Neurology, $3^{\text {rd }}$ Ed, New York, McGraw Hill, 1985;54.

04. Azar I, Karambelkar DJ, Lear E - Neurologic state and psychomotor function following anesthesia for ambulatory surgery. Anesthesiology, 1984;60:347-349.

05. Fiacchino F, Gemma M, Bricchi M et al - Neurological examination in patients recovering from general anesthesia. Ital J Neurol Sci, 1992;13:749-753.

06. Shimoji K, Fujioka H, Fukazawa T et al - Anesthetics and excitatory / inhibitory responses of midbrain reticular neurons. Anesthesiology, 1984;61:151-155.

07. Masahiro I, Sato T, Nishikawa T et al - Hyperlocomotion during recovery from isoflurane anesthesia is associated with increased dopamine turnover in the nucleus accumbens and striatum in mice. Anesthesiology, 1997;86:464-475.

08. Horn EP, Ramsperger K, Sessler DI et al - Shivering following normothermic desflurane or isoflurane anesthesia. Anesthesiology, 1997;87:A321.

09. Sessler DI, Israel D, Pozos RS et al - Spontaneous post-anesthetic tremor does not resemble thermoregulatory shivering. Anesthesiology, 1988;68:843.

\section{RESUMEN}

Soares LF, Helayel PE, Oliveira Filho GR, Amaral R - Alteraciones Transitorias del Examen Neurológico Durante el Despertar de la Anestesia con Enflurano, Isoflurano o Sevoflurano

Justificativa y Objetivos - Anormalidades transitorias del examen neurológico ocurren durante el despertar de la anestesia con halotano, enflurano e isoflurano. Poco se conoce sobre la ocurrencia de anormalidades del examen neurológico durante la recuperación de la anestesia con sevoflurano. Este estudio tuvo como objetivo comparar la prevalencia de tal comportamiento durante la recuperación de la anestesia con enflurano (Grupo E), isoflurano (Grupo I) y sevoflurano (Grupo S).

Método - Fueron estudiados 44 pacientes que recibieron anestesia con enflurano, isoflurano o sevoflurano en $\mathrm{N}_{2} \mathrm{O}$ a $50 \%$. Fueron anotados antes de la inducción, inmediatamente después de la cesación de la administración del anestésico y 5 , 10,15, 20, 30 e 40 minutos después: temperatura timpánica, nivel de consciencia, tono muscular, reflejos pupilar, ciliar, bicipital, patelar y cutaneo-plantar, bien como la ocurrencia de calofríos.

Resultados - Las respuestas de los reflejos pupilar, ciliar, patelar y cutaneo-plantar se correlacionaron con el nivel de consciencia. Los grupos no difirieron cuanto a la prevalencia de hipertonia muscular, hiperreflexia bicipital, clonus plantar y respuesta extensora cutaneo-plantar. Hiperreflexia patelar fue mas frecuente en el grupo del enflurano de que en el grupo del isoflurano. Calofríos fueron mas frecuentes en los grupos $E$ e I de que en el grupo del sevoflurano. La temperatura timpánica no difirió entre los pacientes que presentaron o no calofríos.

Conclusiones - Alteraciones reversibles del examen neurológico pueden estar presentes por hasta 40 minutos durante la recuperación de la anestesia con enflurano, isoflurano o sevoflurano. 